Spray Steelmaking, Ltd. Two full scale experimental units have been set up, at the Lancashire Steel works at Irlam, and at Shelton Iron and Steel Co. At the experimental unit operated by BISRA at Sheffield, the control of the process has been improved. Seventy per cent of the steels in the range 0.6 to 1.4 per cent carbon are produced to within \pm 0.04 per cent of specification. Irons with up to $1 \cdot 0$ per cent phosphorus and 0.12 per cent sulphur have been converted to low carbon steel with acceptable contents of these elements.

In the mechanical working division, successful work has been carried out on a novel method of heating strip or wire. The heating is done by an electrical discharge between the wire and an electrolyte. The difficulty is to maintain a sufficiently stable discharge to produce uniform heating, but the report says that this has been overcome. A direct current source of about 100 volts supplies the energy to a flowing electrolyte, and wire can be heated at a rate of $30 \mathrm{ft} /$ minute, with a claimed heating rate of $4,000{ }^{\circ} \mathrm{C}$ per second. Efficiency of the process is about 30-35 per cent, but could probably be improved; even at this stage, however, BISRA believes the process is attractive for production of stainless steel wire, and discussions for exploitation are under way.

\section{No Helium Here}

THE hope that natural gas from the North Sea might provide the bonus of a European supply of helium seems to be dwindling. The average amount of helium in the strikes that have been made so far at the Leman and West Sole banks is only about 0.02 per cent. This means that unless the Gas Council decides to increase greatly the volume of its liquid gas storage tanks and liquefaction plant, instead of piping the gas ashore and directly into the grid, it will not be economic to try to recover the helium. It seems likely, therefore, that all the helium used in Britain will continue to be imported from North America. At present the two chief suppliers ship helium in cylinders, although there have been experiments with shipping liquid helium from wells in Saskatchewan and Texas, where the natural gas contains up to 2 per cent helium. Although transport costs account for about three-quarters of the price of helium in Britain, for the present at least it appears to be uneconomic to recover helium from natural gas unless it constitutes more than about 0.5 per cent of the total or unless the operation is on a very large scale.

The situation, of course, may change if the world demand for the gas increases greatly-recent developments in cryogenics make some increase in demand inevitable-and shows signs of outstripping supply. This might happen, because apart from the North American sources the only other supplies are a well in South Africa and one in Russia which meets East European demands. Moreover, there have been pessimistic suggestions that the US wells might run dry in the $1980 \mathrm{~s}$. But until there are clear signs of a shortage it is unlikely that North Sea gas will become a source of helium.

\section{Nucleation of a Society}

SINGLE crystals have long been necessary both for academic research and for device applications. The demand for good quality crystals has grown very rapidly with the increasing use of solid state devices such as transistors, integrated circuits and lasers. In spite of this demand, the growth of single crystals has been regarded as a Cinderella subject for many years, particularly as it does not lie within any of the established scientific disciplines. Recently crystal growth has become recognized as a subject worthy of study as a science, and the first international conference on crystal growth was held at Boston, USA, in 1966. Regular national meetings on crystal growth are now held in the USA and the Soviet Union.

The first meeting of British crystal growers was held at Imperial College on April 19 under the chairmanship of Dr E. A. D. White, of the Electrical Engineering Department. The meeting was attended by about sixty crystal growers, from as far afield as Edinburgh and Dublin, who heard five speakers on recent developments in crystal growth techniques. After a broad review by Dr White which included recent theoretical advances, Dr B. A. Smith (University of Sheffield) surveyed the field of growth from the melt with particular emphasis on the are transfer process. There were two papers on growth from aqueous solutions; F. W. Webster (I.R.D., Newcastle) spoke on improvements in hydrothermal equipment, and Dr D. Bloor (Q.M.C., London) on the use of gels to control the rate of diffusion of two interacting solutes. Dr J. M. Robertson (Portsmouth College of Technology) described recent experiments on flux growth in controlled atmosphere, in particular the effect of oxygen pressure on the ferromagnetic resonance linewidth of ferrite crystals. The success of the meeting was evident from the liveliness of the ensuing discussion.

It is intended to hold informal meetings of this nature three times a year, and ultimately to create a formally constituted Crystal Growth Society. However, the next national meeting will not take place until December of this year, because the second International Conference on Crystal Growth will be held in July at the University of Birmingham.

\section{Marriage Bureau for Animals}

A NEw committee, known as the Conservation and Breeding Committee, has been set up by the Federation of Zoological Gardens of Great Britain and Ireland. One of its aims will be to act as a marriage bureau for all zoo animals. It will keep a register of unmated animals (whether rare or not), and it will encourage member zoos to find unpaired specimens. At present twenty-seven zoos are members of the federation, out of a total of about seventy-four zoos in the country. On application for membership of the federation, all zoos are inspected by an independent panel including a veterinary surgeon. They are only accepted if they reach the standards laid down by the federation. So far thirty have applied since 1966, when the federation was established, and all but three have been accepted.

Already there have been some exchanges of animals between zoos for breeding purposes. The London Zoo, for example, has loaned a male bobcat to Twycross Zoo where there are two females, and a male pygmy hippo from Chester Zoo has gone to Whipsnade where there are four females. Although the bureau is intended primarily to help member zoos in breeding 
programmes, non-members are not precluded from taking part in exchanges. International exchanges will no doubt be arranged, too, and the London Zoo has made progress in this direction.

The committee will also support efforts of member zoos towards conservation of species in a wider sense. It will keep a register of all rare mammals and birds kept by member zoos with special emphasis on those species considered to be in danger of extinction in the wild. Zoos will be encouraged to make every effort to breed the rare ones in captivity so that in the future there should be less demands on the depleted wild populations. In some cases zoo-bred animals may be used to reinforce those wild populations which are in danger of complete extinction. This means where practicable the controlled release of captive bred specimens in suitable reserves in their native land. Eagle owls have been sent to Sweden from the Norfolk Wildlife Park for release to augment the native population. This type of co-operation can have beneficial side-effects. For example, thirty Swinhoe's pheasant were sent from Britain to Taiwan where they were released. The Taiwan Government became interested in the project, and as a result it has given twenty-seven species legal protection in the country. Member zoos have in their collections ninety-three species of mammals and thirty-two species of birds totalling 1,194 specimens which are either on the IUCN/ICPB Red Data Book list of endangered species or which are considered likely to become rare in the wild in the future. Among rare species being bred in member zoos are the chimpanzee, the Formosan Sika deer, the Arabian gazelle and several species of pheasant.

\section{Cholera and WHO}

\section{from our Microbiology Correspondent}

THe unexpected spread from the Celebes in the early 1960s of the El Tor cholera vibrio in epidemic proportions reawakened concern and interest in cholera, a disease which, in the previous fifteen years, had shown signs of declining. A collaborative programme on cholera research was established by a joint PhilippinesJapan-WHO Cholera Committee, the chief aims of which were to evaluate the effectiveness of various cholera vaccines and to gain information on cholera carriers, the survival of the causal vibrio in natural environments and the value of various types of therapy. The results of this large scale study, published in the current issue of the Bulletin of the World Health Organization $\left(3^{1 \%}, 697 ; 1967\right)$, represent a major contribution to the understanding of this particular communicable disease.

The first two reports discuss the results of cholera vaccine preparation and field trial. Azurin and his colleagues conducted controlled field trials in the Philippines and found that a single vaccination with routine fluid vaccines (either classical Vibrio cholerae or El Tor) produced only short termed immunity of about two months. This contrasted with a reasonably high protection conferred by a $V$. cholerae oil-adjuvant vaccine for a duration of six months. The disadvantage of the oil-adjuvant vaccine, however, was its propensity to cause severe vaccination reactions in a significant proportion of the population and consequently its use was not recommended. If cholera is to be controlled primarily through vaccination programmes, further assessment of existing and new preparations is imperative. A group at the Serum Institute in Chiba (Ogonuki, Hashizume and Takashashi) discuss the results of their work on oil-adjuvant vaccines. Laboratory tests of this type of vaccine indicated high potency. while volunteer trials confirmed the enhanced protec. tive effect. Unfortunately, the vaccine reaction also was confirmed and found to be caused by local hypersensitivity and foreign-body reactions. Until quite recently it was widely held that antibiotic therapy could not affect cholera prognosis. Subsequently, the validity of chemotherapy as a supplementary treatment for cholera was recognized and has been used to arrest diarrhoea and reduce the incidence of vibrios excreted in the faeces. Kobari et al. investigated the effect of tetracycline, chloramphenicol, erythromycin and kanamycin, administered orally or intravenously. on the course of El Tor cholera. Antibiotic therapy produced a dramatic reduction in the vibrio count in stools within one hour, and after ten hours a complete disappearance of vibrios had occurred. Antibiotics such as kanamycin which are not absorbed were the least effective in treating the disease, even when doses were frequent; this results reflects the short residence time of the drug in the intestines of cholera patients. The intravenous injection of adsorbable antibiotics may prove to be the most efficient since, in the cholera condition, large volumes of body fluids are passed into the intestines and the drug may be present at its site of action in concentrations higher than those resulting from oral administration. The results of case histories appear to substantiate this latter suggestion.

The joint Cholera Committee also investigated cholera carriers in the Philippines. A carrier rate of nearly 22 per cent was found among household contacts of cholera patients, 8 per cent in immediate neighbours but only one-third per cent in the general population. The carrier state appeared to last from five to nineteen days and vibrio strains found in such carriers were identical with strains isolated from chronic sufferers. Perhaps the most significant finding in this study was that of a long-term cholera carrier ("Cholera Dolores"), the first ever to be described. The carrier contracted El Tor cholera in 1962 and continued intermittent excretion of the vibrio up to the time that Azurin and his co-workers reported in the WHO Bulletin. Strain characteristics of the vibrio and immunological evidence confirmed that this was a case of a chronic carrier and not of reinfection. In this instance the causal bacterium produced a reservoir in the gall bladder and/or biliary tract. The authors point out that although "Cholera Dolores" has not been incriminated epidemiologically - almost certainly reflecting the immunity of her family and contacts-the impact of her presence in a non-endemic area could be vastly different. Not unreasonably the analogy is drawn with the classic chronic carrier "Typhoid Mary". Also important in this context are the findings from basic and applied studies on El Tor viability and the occurrence of drug-resistant strains. Prompt and thorough disinfection of contaminated materials is clearly necessary in view of the long period of survival of El Tor vibrios under suitable conditions. Similarly, the discovery of a natural multiple-antibiotic-resistant strain that could transmit its resistance via conjugation to sensitive enteric bacteria such as Salmonella and Shigella must be held up as a further caution to indiscriminate chemotherapy. 